Bull. Korean Math. Soc. 52 (2015), No. 2, pp. 363-366

http://dx.doi.org/10.4134/BKMS.2015.52.2.363

\title{
TWO APPLICATIONS OF LEWIS' THEOREM ON CHARACTER DEGREE GRAPHS OF SOLVABLE GROUPS
}

\author{
Liguo He, Yuanhe Zhao, And Jianxing Bi
}

\begin{abstract}
In this note, we prove Gluck's conjecture and Isaacs-NavarroWolf Conjecture are true for the solvable groups with disconnected graphs by using Lewis' group structure theorem with respect to the disconnected character degree graphs.
\end{abstract}

\section{Introduction}

For a finite group $G$, we attach a graph $\Delta(G)$ to $G$, whose vertices are prime divisors of degree of some nonlinear irreducible complex character of $G$, and distinct vertices $p, q$ are connected when $p q$ divides degree of some irreducible character of $G$.

By $[8,9]$, the number of connected components is at most 3 for any finite groups and 2 for any solvable group. When the graph $\Delta(G)$ is disconnected for the solvable group $G$, P. Palfy described in [12] the structure of $G$; furthermore M. Lewis characterized in [7] the structure of $G$ as follows:

Theorem (Lewis). For a solvable group $G$, the graph $\Delta(G)$ is disconnected if and only if $G$ is one of the groups as Examples 2.1-2.6 in [7].

In [7], Examples 2.1-2.6 along with corresponding Lemmas 3.1-3.6 give detailed information of structure and character degrees of $G$, and even the specific group examples in individual cases.

In this note, we intend to apply the theorem to two open problems of character theory of finite solvable groups. We write $b(G)$ for the largest irreducible character degree of $G$. Gluck conjectured [3] that the inequality $|G: F(G)| \leq b(G)^{2}$ is true for solvable group $G$. Some results have been achieved on the conjecture. For example, Espuelas [1] proved that this inequality holds for odd order groups. We prove the following result.

Received March 31, 2013; Revised June 24, 2013.

2010 Mathematics Subject Classification. Primary 20C15.

Key words and phrases. solvable group, character, fitting subgroup, non-vanishing element.

Project supported by NSF of China (No.10771172) and by NSF of Liaoning Education Department (No.2014399). 
Theorem A. Suppose that $G$ is a finite solvable group such that $\Delta(G)$ is disconnected. Then $|G: F(G)| \leq b(G)^{2}$.

Label $\operatorname{Irr}(G)$ as the full set of complex irreducible characters of $G$. Pick $\chi \in \operatorname{Irr}(G)$. If $g \in G$ satisfies $\chi(g) \neq 0$, then $g$ is said to be a non-vanishing element of $\chi$; further if $g$ is a non-vanishing element for all members of $\operatorname{Irr}(G)$, then $g$ is said to be a non-vanishing element of $G$. In [6], it is conjectured that all non-vanishing elements of a finite solvable group $G$ lie in its Fitting subgroup $F(G)$. This assertion was referred to as Isaacs-Navarro-Wolf Conjecture in [11]. We use $\mathscr{V}(G)$ to denote the subgroup generated by all nonvanishing elements of $G$, i.e., $\mathscr{V}(G)=\langle g| \chi(g) \neq 0$, all $\chi \in \operatorname{Irr}(G)\rangle$, which is called in [4] the strongly vanishing-off subgroup of $G$. Expressed in terms of $\mathscr{V}(G)$, this conjecture equivalently asserts that the inequality $\mathscr{V}(G) \leq F(G)$ is valid for solvable group $G$. A plenty of results are achieved in [6]. For instance, it was proved in $[6$, Theorem D] that the images of nonvanishing elements modulo $F(G)$ are of 2-power order, which implies that the conjecture is true for groups of odd order. In this note, we prove the following result.

Theorem B. Assume that $G$ is solvable so that $\Delta(G)$ is disconnected. Then $\mathscr{V}(G) \leq F(G)$.

The notation and terminology are standard, and we refer readers to $M$. Isaacs book [5] for general background on character theory.

\section{Results}

Lemma 2.1. Let $G$ be a finite group and $V$ a $G$-module, and let $\widetilde{V}=\operatorname{Irr}(V)$. Then $\widetilde{V}$ is a natural $G$-module induced by the action of $G$ on $V$. In particular, $V$ is a completely reducible and faithful $G$-module if and only if $\widetilde{V}$ is.

Proof. Immediate from Lemma 1 of [13].

Proof of Theorem A. We will apply Lewis' theorem. If Example 2.1 holds, then part (iii) of the corresponding Lemma 3.1 yields $G$ has an irreducible character degree $|G: F(G)|$, the desired result follows. If Example 2.2 is true, then $|G: F(G)| \leq\left|S L_{2}(3)\right|$. Note that $\left|S L_{2}(3)\right|=24$. The corresponding Lemma 3.2 (ii) shows $8 \in \operatorname{cd}(G)$, and so $|G: F(G)| \leq 64$, as desired. Example 2.3 shows that $|G: F(G)| \leq\left|G L_{2}(3)\right|$. Note that $\left|G L_{2}(3)\right|=48$. The corresponding Lemma 3.3(ii) shows $16 \in \operatorname{cd}(G)$, obviously $|G: F(G)| \leq 256$, as wanted. If Example 2.4 is valid, then $G=V \rtimes H$ and $Z=C_{H}(V) \leq \bar{Z}(G)$. Let $K=F(H)$ and $E / F(G)=F(G / F(G))$. By the proof of the corresponding Lemma 3.4, we see that $|G: F(G)|=|H: Z|=|H: K||K: Z|$ and $|K: Z|=|E: F(G)|$. The corresponding Lemma 3.4(v) shows $|H: K|$ and $|E: F(G)|$ lie in $\operatorname{cd}(G)$, yielding the result. When Example 2.5 holds, we have $G=Q \rtimes K$ a semidirect product of $K$ coprimely acting on a 2-group $Q, Z=C_{K}(Q)$ is central in $G$ and $|K / Z|=2^{a}+1$. Clearly $|G: F(G)| \leq|K: Z|$. The corresponding Lemma 3.5 indicates $2^{a}+1 \in \operatorname{cd}(G)$, yielding the result. When Example 2.6 is true, 
the corresponding Lemma 3.6 shows $G$ has a normal Sylow $p$-subgroup $P$ and $F(G) / P^{\prime}=F\left(G / P^{\prime}\right)$, furthermore $G / P^{\prime}$ satisfies the hypotheses of Example 2.4 , and thus $|G: F(G)|=\left|G / P^{\prime}: F\left(G / P^{\prime}\right)\right| \leq b\left(G / P^{\prime}\right)^{2} \leq b(G)^{2}$, the desired result follows. The proof is complete.

Proof of Theorem B. We will apply Lewis' theorem. If Example 2.1 is true, then $G$ is a semidirect product $P \rtimes K$ where $P$ is a normal Sylow $p$-subgroup and $K$ is an abelian $p$-complement for some prime $p$, and then $G / F(G)$ is abelian. Gaschütz's theorem (1.12 of [10]) yields $F(G) / \Phi(G)$ is a faithful and completely reducible $G / F(G)$-module (of possibly mixed characteristic). Applying Lemma 2.1 above, we get $G / F(G)$ has regular orbit in $\operatorname{Irr}(F(G) / \Phi(G)$ ), and so there is an irreducible character $\lambda$ of $F(G) / \Phi(G)$ inducing irreducibly to some $\chi \in$ $\operatorname{Irr}(G)$. Therefore, we conclude that all non-vanishing elements of $G$ lie in $F(G)$.

If Example 2.2 holds, then $G=P \rtimes H$, where $P$ is an elementary abelian group of order 9 and $H / Z \cong S L_{2}(3)$ for $Z=C_{H}(P) \leq Z(H)$, moreover $P$ is naturally acted on by $H / Z$ as the special linear group $S L_{2}(3)$. Using GAP [2], we may obtain the character table of $P \rtimes(H / Z)$, which shows $P \rtimes(H / Z)$ has only a single class of non-vanishing elements, and the order of the representative $g Z$ is 3 . Note that $Z$ is central in $G, F(G / Z)=F(G) / Z$ and even $F(G) / Z \cong P$ by Lemma 3.2 of [7]. Obviously $G / Z \cong P \rtimes(H / Z)$. If $g Z \notin F(G / Z)$, then the order of $g F(G)$ in $G / F(G)$ is also 3, which contradicts Theorem D of [6] (see Introduction). Thus $g Z \in F(G / Z)$ and $g \in F(G)$. Note that the non-vanishing elements of $G$ are also the non-vanishing elements of its any quotient.

For Example 2.3, using the argument similar to that in situation of Example 2.2 , the desired result follows.

When Example 2.4 is valid, the corresponding Lemma 3.4 of [7] shows that $G / F(G)$ is metacyclic. Thus $G$ is nilpotent-by-metabelian, and the desired result follows from Theorem 1.1 of [4].

When Example 2.5 is true, we know $G=Q \rtimes K$ where the normal subgroup $Q$ is a non-abelian 2-group and $K$ is an abelian 2-complement. Using the same argument as in situation of Example 2.1, the desired result follows.

If the final Example 2.6 holds, then the corresponding Lemma 3.6 of [7] indicates that $G$ has a normal Sylow $p$-subgroup $P, F(G) / P^{\prime}=F\left(G / P^{\prime}\right)$, and $G / P^{\prime}$ satisfies the hypotheses of Examples 2.4. Thus applying the result of the above situation of Example 2.4, we deduce that $\mathscr{V}\left(G / P^{\prime}\right) \leq F(G) / P^{\prime}$. Also $\mathscr{V}(G) P^{\prime} / P^{\prime} \leq \mathscr{V}\left(G / P^{\prime}\right)$, we conclude $\mathscr{V}(G) \leq F(G)$, as desired. The proof is finished.

\section{References}

[1] A. Espuelas, Large character degrees of groups of odd order, Illinois J. Math. 35 (1991), no. 3, 499-505.

[2] The GAP Group, GAP-Groups, algorithms, and programming, version 4.5, http:// www.gap-system.org, 2012. 
[3] D. Gluck, The largest irreducible character degree of a finite group, Canad. J. Math. 37 (1985), no. 2, 442-451.

[4] L. G. He, Notes on non-vanishing elements of finite solvable groups, Bull. Malays. Math. Sci. Soc. (2) 35 (2012), no. 1, 163-169.

[5] I. M. Isaacs, Character Theory of Finite Groups, Academic Press, New York, 1976.

[6] I. M. Isaacs, G. Navarro, and T. R. Wolf, Finite group elements where no irreducible character vanishes, J. Algebra 222 (1999), no. 2, 413-423.

[7] M. Lewis, Solvable groups whose degree graphs have two connected components, J. Group Theory 4 (2001), no. 3, 255-275.

[8] O. Manz, Degree problems II: $\pi$-separable character degrees, Comm. Algebra 13 (1985), no. 11, 2421-2431

[9] O. Manz, R. Staszewski, and W. Willems, On the number of components of a graph related to character degrees, Proc. Amer. Math. Soc. 103 (1988), no. 1, 31-37.

[10] O. Manz and T. R. Wolf, Representations of Solvable Groups, Cambridge Univ. Press, Cambridge, 1993.

[11] A. Moretó and T. R. Wolf, Orbit sizes, character degrees and Sylow subgroups, Adv. Math. 184 (2004), no. 1, 18-36.

[12] P. P. Pálfy, On the character degree graph of solvable groups. II: disconnected graphs, Studia Sci. Math. Hungar. 28 (2001), 339-355.

[13] J. P. Zhang, A note on character degrees of finite solvable groups, Comm. Algebra 28 (2000), no. 9, 4249-4258.

LiguO HE

Department of Mathematics

Shenyang University of Technology

Shenyang 110870, P. R. China

E-mail address: cowleyhe@126.com

YuANHE ZHAO

Department of Mathematics

Shenyang University of TECHNOLOGY

Shenyang 110870, P. R. China

JIANXING BI

Department of Mathematics

LIAONING UNIVERSITY

Shenyang 110036, P. R. China

E-mail address: mabjx@sina.com 